\title{
LETTER
}

\section{Clinicolaboratory study of 25 fatal cases of COVID-19 in Wuhan}

\author{
Wen-Jun Tu${ }^{1}$, Jianlei $\mathrm{CaO}^{2}$, Lei Yu ${ }^{3}$, Xiaorong $\mathrm{Hu}^{2^{*}}$ and Qiang Liu ${ }^{1 *}$ (])
}

(c) 2020 Springer-Verlag GmbH Germany, part of Springer Nature

\section{Dear Editor,}

In December 2019, cases of pneumonia associated with a novel 2019 coronavirus emerged in Wuhan (Hubei Province, China) [1]. The causative agent was subsequently named SARS-CoV-2 and the resulting disease COVID19. As of 17 March 2020, 80,894 cases of COVID-19 were reported in China of which 3237 (4\%) were fatal. In Wuhan, $2490(4.98 \%)$ of 50,005 cases died. In addition, 98,218 cases from 104 countries outside of China were laboratory-confirmed, of which 4189 (4.27\%) were fatal. A previous study suggested that the condition of $11(11 \%)$ patients worsened in a short period of time and they died of multiple organ failure [2], while Wang et al. [3] reported that $4.3 \%$ of COVID-19 cases were fatal. A national study of 1099 patients with COVID-19 found that 55 patients $(5 \%)$ were admitted to an intensive care unit and 15 (1.36\%) succumbed to the infection [4]. It is important to emphasize that most patients studied previously were hospitalized and thus the full spectrum of COVID-19 severity is still being elucidated [1-4]. We aimed to further explore the clinicolaboratory characteristics, hospital complications and treatments of 25 fatal cases of COVID-19. The clinicolaboratory characteristics of survivors $(N=149)$ and non-survivors were also compared.

This was a single-center retrospective analysis. All consecutive fatal cases of COVID-19 admitted to Wuhan University Zhongnan Hospital from 3 January to 24 February 2020 were included. COVID-19 was confirmed

\footnotetext{
*Correspondence: cjlzn14@whu.edu.cn; liuqiang@irm-cams.ac.cn ${ }^{1}$ Institute of Radiation Medicine, China Academy of Medical Science and Peking Union Medical College, No. 238, Baiti Road, Tianjin 300192, People's Republic of China

2 Department of Cardiology, Zhongnan Hospital, Wuhan University, No. 169, Donghu Road, Wuhan 430071, People's Republic of China

Full author information is available at the end of the article

Wen-Jun Tu and Jianlei Cao have contributed equally to this work.
}

using throat swab samples by real-time RT-PCR $[3,5]$. Epidemiological, clinical and laboratory data as well as information on treatments received, hospital complications and causes of death were collected. Blood samples were collected at admission. The study was approved by the Ethics Committee of Wuhan University Zhongnan Hospital and informed consent was waived by the Ethics Committee.

Twenty-five fatal COVID-19 cases were included. The median age of these patients was 70 years (interquartile range [IQR] 64-80 years) and 19 (76\%) were men. The median time from onset of symptoms to hospital admission and death was 7 days (IQR 1-10 days) and 19 days (IQR 13-26 days), respectively. As shown in Table 1, fatal cases were older (70 years, IQR $64-80$ years vs. 51 years, IQR $37-62$ years), disproportionately male (76\% vs. $40.3 \%)$ and more often suffered from comorbidities (64\% vs. 24.2\%; cardiovascular and cerebrovascular diseases: $32 \%$ vs. $7.4 \%$ ) compared with non-fatal cases. Fatal cases were also more likely to be admitted to intensive care units (36\% vs. $10.7 \%$ ) and had higher medical expenses (53,745 CNY, IQR 30,286-112,268 CNY vs. 14,507 CNY, IQR 8813-27,617 CNY).

During the study period, 174 patients (all COVID19-positive hospital admissions) had an outcome (death or discharge). Thus, the case fatality rate was 14.4\% (95\% CI 9.2-19.6\%). The most common cause of death was multiple organ dysfunction syndrome (56\%). Cardiac arrest (20\%), respiratory failure (16\%) and acute respiratory distress syndrome (16\%) were other causes of death (Table 1). Acute respiratory distress syndrome (shock), secondary bacterial infection and acute cardiac/kidney/liver injury were common during hospitalization. Most patients were treated with methylprednisolone (76\%), invasive mechanical ventilation (68\%) and oseltamivir (64\%). Fatal cases experienced hospital complications and received aggressive

\section{实


Table 1 Baseline characteristics and laboratory results of included cases of COVID-19

\begin{tabular}{|c|c|c|c|}
\hline & Non-survivors & Survivors & $p$-value \\
\hline N & 25 & 149 & - \\
\hline Age (years) & $70(64-80)$ & $51(37-62)$ & $<0.001$ \\
\hline Sex-male & $19(76.0)$ & $60(40.3)$ & $<0.001$ \\
\hline $\mathrm{BMI}\left(\mathrm{kg} / \mathrm{m}^{2}\right)$ & $24.6(22.3-28.3)$ & $23.6(21.6-25.6)$ & 0.125 \\
\hline Temperature at admission $\left({ }^{\circ} \mathrm{C}\right)$ & $38.1(37.1-39.0)$ & $38.0(37.3-38.9)$ & 0.383 \\
\hline \multicolumn{4}{|l|}{ Comorbidities } \\
\hline Any & $16(64.0)$ & $36(24.2)$ & $<0.001$ \\
\hline Hypertension & $12(48.0)$ & $25(16.8)$ & $<0.001$ \\
\hline Diabetes & $6(24.0)$ & $11(7.4)$ & 0.010 \\
\hline Cardiovascular-cerebrovascular diseases & $8(32.0)$ & $8(7.4)$ & $<0.001$ \\
\hline Respiratory diseases & $4(16.0)$ & $8(7.4)$ & 0.130 \\
\hline \multicolumn{4}{|l|}{ Onset of symptom to (days) } \\
\hline Hospital admission & $7(1-10)$ & $7(3-9)$ & 0.381 \\
\hline Death or discharge & $19(13-26)$ & $20(13-27)$ & 0.894 \\
\hline ICU admission & $9(36.0)$ & $16(10.7)$ & 0.003 \\
\hline Cost of hospitalization (CNY) & 53745 (30 286-112 268) & 14507 (8 813-27 617) & $<0.001$ \\
\hline \multicolumn{4}{|l|}{ Laboratory findings at admission ${ }^{a}$} \\
\hline White blood cell $\left(10^{9} / I\right)$ & $6.88(4.96-13.48)$ & $4.22(3.21-5.91)$ & $<0.001$ \\
\hline Elevated (>9.5) & $10(40.0)$ & $10(6.7)$ & $<0.001$ \\
\hline Lymphocyte $\left(10^{9} / \mathrm{l}\right)$ & $0.53(0.33-0.82)$ & $0.92(0.67-1.23)$ & $<0.001$ \\
\hline Reduced $\left(<1.1 \times 10^{9} / \mathrm{l}\right)$ & $23(92.0)$ & $99(66.4)$ & 0.010 \\
\hline IL-6 (pg/ml) & $108.8(44.1-177.9)$ & $16.8(4.4-76.9)$ & $<0.001$ \\
\hline Elevated (> 2.9 pg/ml) & $25(100.0)$ & $115(77.2)$ & 0.017 \\
\hline D-dimer (ng/ml) & $3306(1790-7512)$ & $660(370-1108)$ & $<0.001$ \\
\hline Elevated (> 500 ng/ml) & $24(96.0)$ & $89(59.7)$ & $<0.001$ \\
\hline $\operatorname{CRP}(\mathrm{mg} / \mathrm{l})$ & $118(22-184)$ & $22(6-45)$ & $<0.001$ \\
\hline Elevated (> 10 mg/l) & $25(100.0)$ & $95(63.8)$ & $<0.001$ \\
\hline \multicolumn{4}{|l|}{ Treatment } \\
\hline Umifenovir & $3(12.0)$ & $70(47.0)$ & 0.001 \\
\hline Oseltamivir & $16(64.0)$ & $61(40.9)$ & 0.032 \\
\hline Lopinavir & $5(20.0)$ & $46(30.9)$ & 0.269 \\
\hline Methylprednisolone & $18(72.0)$ & $71(47.7)$ & 0.024 \\
\hline Noninvasive ventilation & $4(16.0)$ & $5(3.4)$ & 0.031 \\
\hline Invasive mechanical ventilation & $17(68.0)$ & $2(1.3)$ & $<0.001$ \\
\hline ECMO & $3(12.0)$ & $3(2.0)$ & 0.052 \\
\hline CRRT & $7(28.0)$ & $1(0.7)$ & $<0.001$ \\
\hline \multicolumn{4}{|l|}{ Complications } \\
\hline Shock and/or ARDS & $23(92.0)$ & $8(5.4)$ & $<0.001$ \\
\hline Secondary bacterial infection & $20(80.0)$ & $6(4.0)$ & $<0.001$ \\
\hline Acute cardiac injury ${ }^{b}$ & $18(72.0)$ & $7(4.7)$ & $<0.001$ \\
\hline Acute kidney injury & $19(76.0)$ & $8(5.4)$ & $<0.001$ \\
\hline Acute liver injury & $15(60.0)$ & $30(20.1)$ & $<0.001$ \\
\hline \multicolumn{4}{|l|}{ Cause of death } \\
\hline MODS & $14(56.0)$ & - & \\
\hline ARDS & $2(8.0)$ & - & \\
\hline Cardiac arrest & $5(20.0)$ & - & \\
\hline Respiratory failure & $4(16.0)$ & - & \\
\hline
\end{tabular}

The results were presented as median (IQR) for continuous variables and number (\%) for categorical variables. The different characteristics between death and survival groups were tested by Mann-Whitney $U$ test (continuous variables) or Chi-square test (categorical variables). A two-sided a of less than 0.05 was considered statistically significant

ICU intensive care unit, BMI body mass index, CNY, China Yuan, IL-6 interleukin-6, CRP C reaction protein, ECMO extracorporeal membrane oxygenation, MODS multiple 
Table 1 (continued)

organ dysfunction syndrome, CRRT continuous renal replacement therapy, $A R D S$ acute respiratory distress syndrome

a We calculated the average value if one patient had multiple tests

b Acute cardiac injury was diagnosed if serum levels of cardiac biomarkers (e.g., troponin I) were above the 99th percentile upper reference limit, or new abnormalities were shown in electrocardiography and echocardiography

treatment strategies more often than non-fatal cases (Table 1). Interestingly, fatal cases were treated more often with oseltamivir and methylprednisolone, but less often with umifenovir (Table 1).

Serum levels of interleukin-6, C-reactive protein and D-dimer were higher in non-survivors than in survivors, while lymphocyte counts were lower (Table 1, Fig. 1). Nearly, all fatal cases had abnormal coagulation, and 24 (96\%) fatal cases showed elevated D-dimer levels. All fatal cases showed evidence of cytokine abnormalities and establishment of an inflammatory state as demonstrated by elevated interleukin- 6 and C-reactive protein levels.
In summary, COVID-19 mortality is more common in older male patients with comorbidities and is mainly caused by multiple organ dysfunction syndrome. The roles of hypercoagulability and pathological inflammatory states should not be ignored. Similarly, other literature recently published in this population also showed that the increasing odds of in-hospital death associated with older age, the presence of underlying diseases, elevated inflammatory and d-dimer greater than $1 \mu \mathrm{g} / \mathrm{ml}$ on admission $[6,7]$.

An interferon- $\gamma$-related cytokine storm may be involved in immunopathological damage in SARS patients [8]. In addition, SARS patients with early-stage disease, especially those with subsequent poor outcomes,
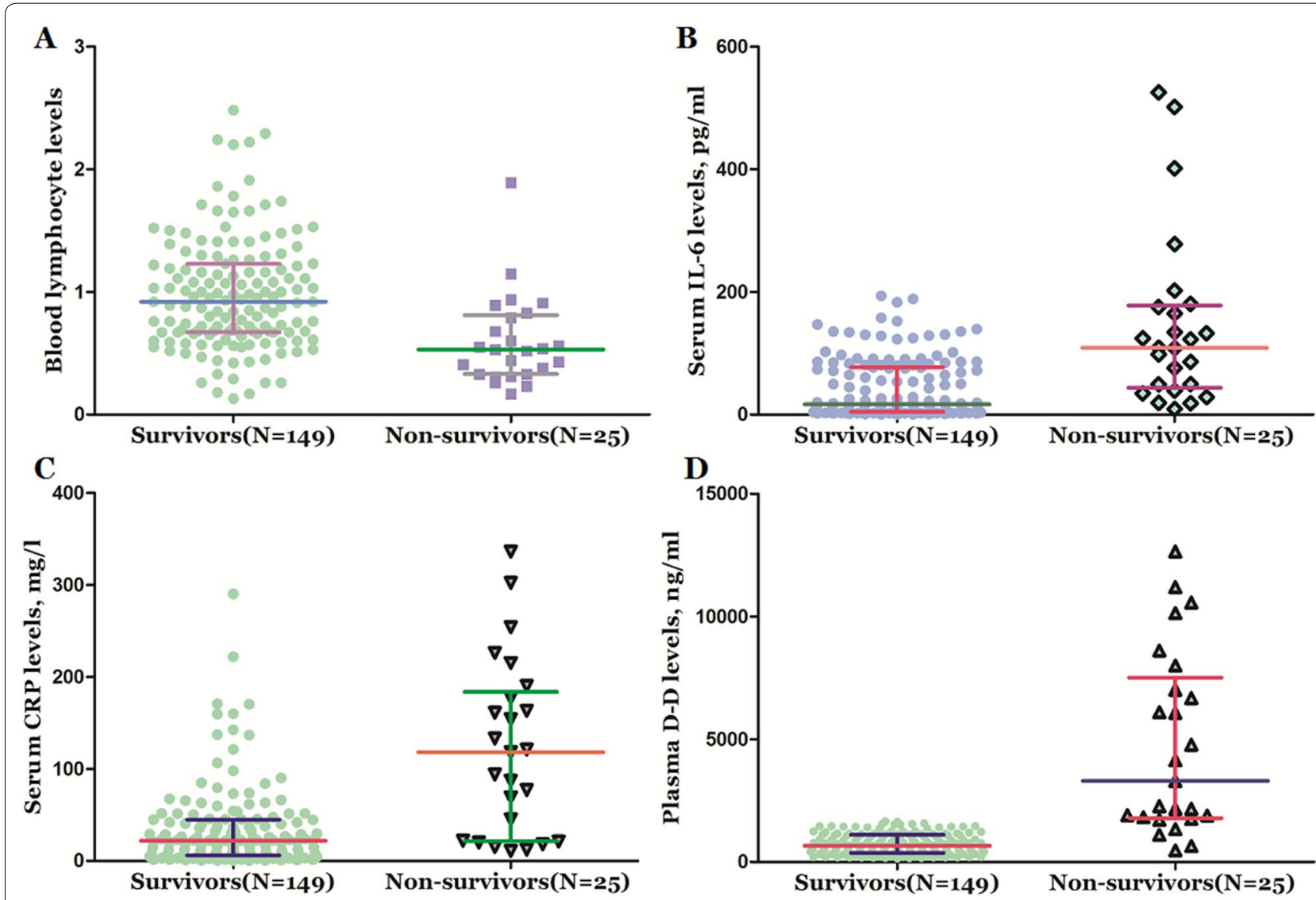

Fig. 1 Blood levels of biomarkers in non-survivors and survivors of COVID-19. a Levels of lymphocyte in non-survivors and survivors; b levels of interleukin-6 in non-survivors and survivors; c levels of $C$ reaction protein in non-survivors and survivors; $\mathbf{d}$ levels of D-dimer in non-survivors and survivors. All data are medians and interquartile ranges (IQR), with dot plots representing all values 
had very high numbers of tumor necrosis factor- $\alpha$ - and interleukin-6-producing cells in the blood [9]. Previous studies also reported that fatal cases of COVID-19 had higher levels of clotting factors and cytokines [1-3, 7]. We speculate that the pathogenesis of fatal cases might involve uncontrolled release of immune mediators (i.e., a 'cytokine storm'). Ruan et al. [6] also suggested that COVID-19 mortality might be due to virus-activated "cytokine storm syndrome" or fulminant myocarditis [6]. Tocilizumab (a monoclonal antibody targeting the interleukin-6 receptor) had been used to treat cytokine storm syndrome [10], and a clinical trial to assess its use in COVID-19 patients has been registered. These findings offer new insights into the characteristics of fatal cases of COVID-19, which may help identify patients at high risk of severe disease or death. The limitations of this study are listed in the supplementary material.

\section{Electronic supplementary material}

The online version of this article (https://doi.org/10.1007/s00134-020-06023-4) contains supplementary material, which is available to authorized users.

\section{Author details \\ ${ }^{1}$ Institute of Radiation Medicine, China Academy of Medical Science and Peking Union Medical College, No. 238, Baiti Road, Tianjin 300192, People's Republic of China. ${ }^{2}$ Department of Cardiology, Zhongnan Hospital, Wuhan University, No. 169, Donghu Road, Wuhan 430071, People's Republic of China. ${ }^{3}$ Department of Infectious Diseases, The Fourth Affiliated Hospital of Harbin Medical University, Harbin, People's Republic of China.}

\section{Acknowledgements}

We thank all patients included in this study. We are really grateful to all the health workers around the world. Their expertise and humanity are fundamental to stop SARS-COV-2 from spreading further. We thank Liwen Bianji, Edanz Editing China (www.liwenbianji.cn/ac), for editing the English text of a draft of this manuscript. We also acknowledge the contribution of the editors and reviewers who have helped us to improve the manuscript.

\section{Author contributions}

Drs W-JT and JC contributed equally as the co-author. Drs XH and QL contributed equally as senior authors. Drs W-JT and JC had full access to all of the data in the study and take responsibility for the integrity of the data and the accuracy of the data analysis. Concept and design were contributed by $\mathrm{JC}, \mathrm{XH}$, LY, W-JT and QL. Acquisition, analysis, or interpretation of data were contributed by JC, XH, LY, W-JT and QL. Drafting of the manuscript was contributed by JC, W-JT and QL. Critical revision of the manuscript for important intellectual content was contributed by XH and LY. Statistical analysis was contributed by $J C$ and W-JT. Administrative, technical, or material support was contributed by JC, XH, W-JT and QL. Supervision was contributed by JC and XH. W-JT and QL obtained funding.

\section{Funding}

This study was supported by funding from CAMS Innovation Fund for Medical Science (Dr. Liu, No. 2017-I2M-1-016; Dr. Tu, 2019-12M-2-006); Natural Science Foundation of Tianjin (Dr. Tu, No. 19JCYBJC26600) and China Postdoctoral Science Foundation funded project (Dr. Tu, No. 2019M660921).

\section{Data availability}

Data available can be obtained from the corresponding author.

Compliance with ethical standards

\section{Conflicts of interest}

None reported.

\section{Consent for publication}

Not applicable.

\section{Role of the funders/sponsors}

The study funders/sponsors had no role in the design and conduct of the study; collection, management, analysis, and interpretation of the data; preparation, review, or approval of the manuscript; and decision to submit the manuscript for publication.

\section{Publisher's Note}

Springer Nature remains neutral with regard to jurisdictional claims in published maps and institutional affiliations.

Accepted: 23 March 2020

Published online: 6 April 2020

\section{References}

1. Huang C, Wang Y, Li X, Ren L, Zhao J, Hu Y et al (2020) Clinical features of patients infected with 2019 novel coronavirus in Wuhan, China. Lancet 395:497-506

2. Chen N, Zhou M, Dong X, Qu J, Gong F, Han Y et al (2020) Epidemiological and clinical characteristics of 99 cases of 2019 novel coronavirus pneumonia in Wuhan, China: a descriptive study. Lancet 395:507-513

3. Wang D, Hu B, Hu C, Zhu F, Liu X, Zhang J et al (2020) Clinical characteristics of 138 hospitalized patients with 2019 novel coronavirusinfected pneumonia in Wuhan. China. JAMA. https://doi.org/10.1001/ jama.2020.1585

4. Guan WJ, Ni ZY, Hu Y, Liang WH, Ou CQ, He JX et al (2020) Clinical characteristics of 2019 novel coronavirus infection in China. N Engl J Med. https ://doi.org/10.1056/NEJMoa2002032

5. Cao JL, Hu XR, Cheng W, Yu L, Tu WJ, Liu Q (2020) Clinical features and short-term outcomes of 18 patients with corona virus disease 2019 in intensive care unit. Intensive Care Med. https://doi.org/10.1007/s0013 4-020-05987-7

6. Ruan $Q$, Yang K, Wang W, Jiang L, Song J (2020) Clinical predictors of mortality due to COVID-19 based on an analysis of data of 150 patients from Wuhan, China. Intensive Care Med 9:9. https://doi.org/10.1007/s0013 4-020-05991-X

7. Zhou F, Yu T, Du R et al (2020) Clinical course and risk factors for mortality of adult inpatients with COVID-19 in Wuhan, China: a retrospective cohort study. Lancet. https://doi.org/10.1016/S0140-6736(20)30566-3

8. Huang KJ, Su IJ, Theron M, Wu YC, Lai SK, Liu CC, Lei HY (2005) An interferon- $\gamma$-related cytokine storm in SARS patients. J Med Virol 75(2):185-194

9. Jones BM, Ma ESK, Peiris JSM, Wong PC, Ho JCM, Lam Lai KN, Tsang KWT (2004) Prolonged disturbances of in vitro cytokine production in patients with severe acute respiratory syndrome (SARS) treated with ribavirin and steroids. Clin Exp Immunol 135:467-473

10. Barrett D (2019) IL-6 blockade in cytokine storm syndromes. In: Cron R, Behrens E (eds) Cytokine storm syndrome. Springer, Cham 